\title{
Molecular simulation study of the specific combination between four kinds of phthalic acid esters and human serum albumin
}

\author{
Zhiwei $\mathrm{Wu}^{\mathrm{a}}$, Zhongsheng $\mathrm{Yi}^{\mathrm{a}, *}$, Lu Dong ${ }^{\mathrm{a}}$, Aiqian Zhang ${ }^{\mathrm{b}}$ \\ a College of Chemistry and Bioengineering, Guilin University of Technology, Guilin 541004, China \\ ${ }^{\mathrm{b}}$ State Key Laboratory of Environmental Chemistry and Ecotoxicology, Research Center for Eco-Environmental Sciences, Chinese Academy of Sciences, \\ Beijing 100085, China
}

\section{A R T I C L E I N F O}

\section{Article history:}

Received 15 August 2015

Received in revised form

17 December 2015

Accepted 19 December 2015

Available online 23 December 2015

\section{Keywords:}

PAES

HSA

Fluorescence spectroscopy

Molecular modeling method

Binding mechanism

\begin{abstract}
A B S T R A C T
The interaction between endocrine disruptor phthalic acid esters (PAEs) and human serum albumin (HSA) was studied by fluorescence spectroscopy and molecular modeling methods. The efficiency of energy transfer and the distance between HSA and PAEs were calculated. The results showed that all of the four kinds of PAEs could quench the intrinsic fluorescence of the HSA, with the mechanisms of static quenching and non-radiative energy transfer. Molecular docking study and thermodynamic analysis revealed that the binding behavior was mainly governed by hydrophobic force. And the results of site marker competitive experiments and modeling method suggested that the four PAEs would mainly bind to the HSA in sub-domain IIIA, which demonstrated that the experimental results could coordinate with the theoretical results. Molecular dynamic simulation (MD) revealed that HSA did have a slight conformational change when it bound with PAEs. It also verified the greater stability of HSA-PAEs complex compared to free protein.
\end{abstract}

(c) 2015 Elsevier B.V. All rights reserved.

\section{Introduction}

Human serum albumin (HSA) contains 585 amino acids, which serves as a transporting agent for various endogenous and exogenous compounds, like fatty acids, nutrients, steroids, certain metal ions, hormones and drugs (Carter et al., 1994; Yamasaki et al., 1996; Pu et al., 2014; Ibrahim et al., 2010). The whole structure is composed of three homologous domains (I, II and III), each could divided into two sub-domains ( $\mathrm{A}$ and $\mathrm{B}$ ), and only one tryptophan residue (Trp-214) in the sub-domain IIA (Deeb et al., 2010). Ligands generally bind with the two principal regions of HSA that are located in the hydrophobic cavities of sub-domain IIA and IIIA, that is, Sudlow's site I and site II, respectively. Site I is located in the hydrophobic cavity of sub-domain IIA which is capable of binding most neutral or heterocyclic compounds by strong hydrophobic interactions, whereas site II in the sub-domain IIIA could bind many aromatic carboxylic acids by Van der Waals force or hydrogen bonding interaction, respectively.

Phthalic acid esters (PAEs) are widely used in toys and personal care products, etc. and they can migrate from the plastic into the external environment, plants (Blair et al., 2009; Mo et al., 2009; Liu

\footnotetext{
* Corresponding author.

E-mail address: yzs@glut.edu.cn (Z.Yi).
}

et al., 2013), which scatter the potential risk for human exposure (Romani et al., 2014). Moreover, PAEs are considered to be a class of environmental endocrine disruptors which could cause testicular effect, influence the hormone synthesis and increase miscarriages or other gestational complications (Qu et al., 2009; Hansen and Grafton, 1994; Suzuki et al., 2001). To investigate the interaction between HSA and PAEs in detail has significant meaning for further understanding the pharma-cokinetics and pharmacodynamic properties of PAEs as it strongly affect the distribution of PAEs and the free fraction that is available for subsequent interactions with targeted receptors. However, few experiment studies of the pollution of phthalate esters in the HSA were reported due to the complexity of the sample substrate, and a detailed mechanism of interaction is still elusive (Xie et al., 2011; Zhou et al., 2012).

In this study, we emphasizes particularly on the mechanism and analysis of the interaction of HSA with four test PAEs (structure shown in Fig. 1) such as dimethyl phthalate (DMP), diethyl phthalate (DEP), dibutyl phthalate (DBP) and dioctyl phthalate (DOP) at physiological buffer ( $\mathrm{pH} 7.4$ ) by fluorescence technique and dynamics approaches. Compared the binding mode site I and site II of HSA and its parameters, the nature of interacting forces were discussed. The molecular level interactions, conformational changes of the protein HSA after binding to the probe, and the flexibility at the binding sites were also explored by molecular docking and MD simulations. 


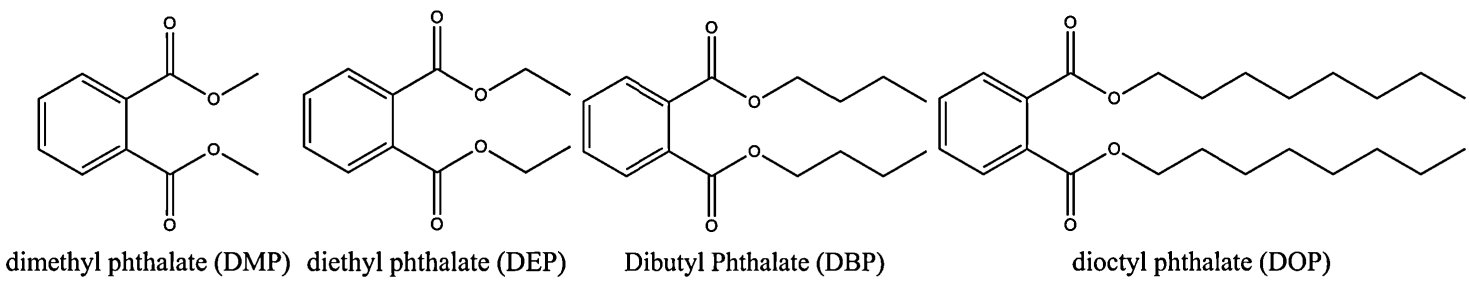

Fig. 1. Structure of DMP, DEP, DBP and DOP.

\section{Materials and methods}

\subsection{Reagents and apparatus}

DMP, DEP, DBP and DOP were obtained from Sino-Pharm Co. Ltd, and the $1.0 \times 10^{-3} \mathrm{M}$ PAEs solution was prepared by dissolving them in ethanol solution, respectively; HSA (98\%) was purchased from Sigma Chemical Company; $1.0 \times 10^{-5}$ M HSA stock solution and $1.0 \times 10^{-3} \mathrm{M}$ warfarin (ibuprofen) stock solution was prepared by dissolving them in $\mathrm{pH} 7.4$ Tris- $\mathrm{HCl}$ buffer solution $(0.2 \mathrm{M}$, containing $0.15 \mathrm{M}$ of $\mathrm{NaCl}$ ). The stock solution was stored at $-4{ }^{\circ} \mathrm{C}$ before assay. All of the reagents were analytical grade and the water used was doubly distilled water.

RF-5301PC fluorescence spectrometer with supporting equipments such as thermostatic water bath, heated pool and $1.0 \mathrm{~cm}$ quartz cuvette (Shimadzu, Japan); Cary-50 UV-visible absorption spectra spectrophotometer (Varian, USA); EL204 electronic analytical balancer (Mettler-Toledo Instruments Co., Ltd); PT-10 portable pH meter (Sartorius, Germany).

\subsection{Spectral measurements}

In a typical fluorescence measurement, $1.0 \mathrm{~mL}$ HSA stock solution and $2.00 \mathrm{~mL} \mathrm{pH} 7.4$ Tris- $\mathrm{HCl}$ buffer solution were added to a $10.0 \mathrm{~mL}$ test tube. The PAEs solution was then gradually added to the tube using a trace syringe. The fluorescence emission spectra were measured at $291 \mathrm{~K}, 298 \mathrm{~K}$ and $310 \mathrm{~K}$, respectively by a thermostatic bath to maintain the temperatures. The width of the excitation and emission slits was set at 3.0 and $5.0 \mathrm{~nm}$, respectively. $280 \mathrm{~nm}$ was chosen as the excitation wavelength and the emission wavelength was recorded from 300 to $500 \mathrm{~nm}$.

Synchronous fluorescence spectra of HSA in the absence and presence of the gradually increasing amount of PAEs was examined with constant differences of $\Delta \lambda=60 \mathrm{~nm}$ and $15 \mathrm{~nm}$ between the excitation and emission fluorescence monochromators, respectively. Site marker competitive experiments were carried out using Warfarin and Ibuprofen as site markers with fluorescence titration methods. The concentrations of HSA and Warfarin/Ibuprofen were all stabilized at $1.0 \times 10^{-6} \mathrm{M}$. PAEs were then gradually added to the HSA-Warfarin and HSA-Ibuprofen solutions. An excitation wavelength of $280 \mathrm{~nm}$ was selected and the fluorescence spectra were recorded in the range of $300-500 \mathrm{~nm}$.

\subsection{Molecular docking}

The following molecular docking study was conducted to explore possible binding positions between PAEs and HSA. The initial structures of all the molecules were generated by molecular modeling software SYBYL-X 1.1. Surflex-Dock program is used for molecular docking. Water molecules were removed and hydrogen atoms were added to the proteins, and Kollman Uni charges were also assigned to HSA. All other parameters were maintained at their default settings. The known crystal structure of HSA (PDB ID:1N5U, Wardell et al., 2002) was obtained from the Brookhaven Protein Data Bank (http://www.rcsb.org/pdb). The structure geometry was optimized using the Tripos force field with a steepest descent method, followed by conjugate gradient in $10^{4}$ steps, and a convergence criterion for the energy gradient of $0.05 \mathrm{KJ} /(\mathrm{mol} \AA)$. Finally, the highest-scored conformation was selected as the final bioactive conformation

\subsection{Molecular dynamics simulations}

A 10 ns molecular dynamics simulation of the complex was carried out with GROMACS 4.5.5 package by using the GROMOS96 43A1 force field. The topology parameters of PAEs were built by using PRODRG (http://davapc1.bioch.dundee.ac.uk/cgibin/prodrg/). Then the complex was immersed in a cubic box of extended single point charge (SPC) water model. The solvated system was neutralized by adding $14 \mathrm{Na}^{+}$counter ions. To release conflicting contacts, energy minimization was performed using the steepest descent method with 2000 steps. The periodic boundary condition was used and the motion equations were integrated by applying the leaf-frog algorithm with a time step of $2 \mathrm{fs}$. The atomic coordinates were recorded at every 2 ps during the simulation for latter analysis. Finally, the full system was subjected to $10 \mathrm{~ns}$ MD at $300 \mathrm{~K}$ temperature and 1 bar pressure.

\section{Results and discussion}

\subsection{Characteristics of molecular dynamics simulation}

The structural behaviors and energetic features of HSA-PAEs interaction were further analyzed based on the snapshots taken from the molecular trajectory of MD simulation. Root Mean Square Deviation (RMSD) of HSA and PAEs was examined to investigate the stability of the system (protein, ligand, ions, water, etc.). RMSD values for free HSA and HSA-PAEs complexes during 10 ns MD simulations are shown in Fig. 2(a). It is observed that the total energy for all molecular systems remains stable with some fluctuations after $4 \mathrm{~ns}$ of simulation, so all of the following analysis was carried out based on the stability of the simulation after the 4 ns of equilibration. The stability of the whole protein structure makes it possible to obtain reliable analysis on ligand binding and its effect on HSA-PAEs interaction through MD simulation. The relative decrease in RMSD value of the complex with respect to free HSA also indicates the conformational change, increased rigidity and stability of the protein HSA upon binding of PAEs (Ko et al., 2008). By comparing the RMSD of PAEs, it can be stated that site II is more favorable for PAEs binding to HSA.

Protein mobility was also analyzed by calculating the timeaveraged root mean square fluctuation (RMSF) (Jana et al., 2012) of free HSA and HSA-PAEs complexes based on the 10 ns trajectory data (Fig. 2(b)). The general profiles of atomic fluctuations were found to be very similar to each other. The comparison of RMSF for HSA-PAEs in sub-domains IIIA, where PAEs is binding, shows that RMSF value of HSA-PAEs in sub-domains IIIA was much more than free HSA, with very little fluctuation.

The radius of gyration $(R g)$ values of free HSA and HSA-PAEs complexes are shown in Fig. 2(c). In both systems, $R g$ values were 

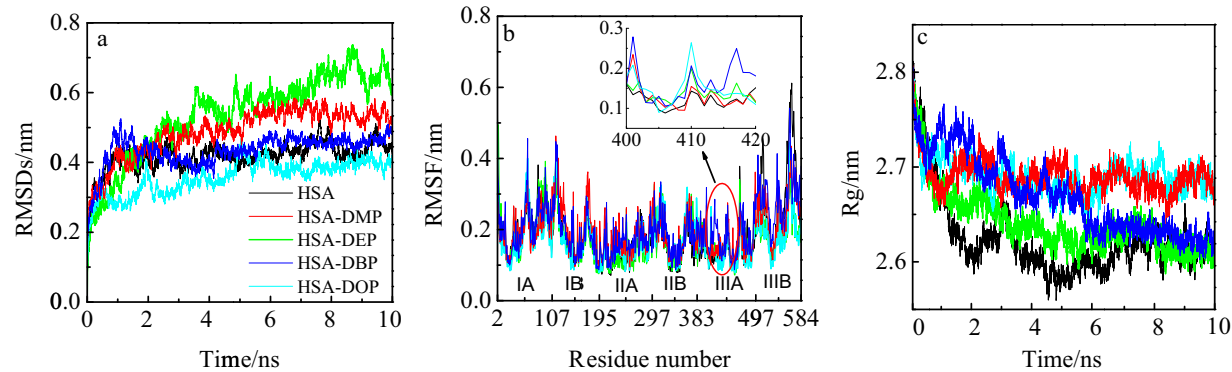

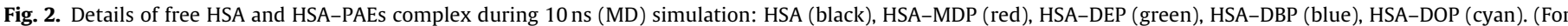
interpretation of the references to color in this figure legend, the reader is referred to the web version of this article.)

stabilized at about $4 \mathrm{~ns}$, indicating that the MD simulation could achieve equilibrium after $4 \mathrm{~ns}$. The radius gyration of both HSA and HSA-PAEs are approximately similar to each other, which clearly indicate that there are moderate conformational changes during the simulation (Li et al., 2010). The MD simulation studies revealed that HSA and HSA-PAEs complexes were stabilized around $4 \mathrm{~ns}$, and the complex also exhibited minor conformational change. The sub-domain IIIA (site II) is predicted as more favorable for PAEs binding.

\subsection{Characteristics of synchronous fluorescence spectra}

Synchronous fluorescence spectrometry has the advantages like high sensitivity, good selectivity and less interference (Jiang et al., 2002). When the $\Delta \lambda$ between excitation wavelength and emission wavelength were stabilized at $15 \mathrm{~nm}$ and $60 \mathrm{~nm}$, the synchronous fluorescence gives the characteristic information of tyrosine residues or tryptophan residues. It can be seen from Fig. 3 that the fluorescence intensity of HSA decreased in the presence of the four PAEs and the maximum emission wavelength has slight blue-shifts. These observations suggest that PAEs have lower effect on the microenvironment of the tyrosine residues in HSA compared with that by tryptophan. In addition, the small shifts in the spectra express the hydrophobicity of both residues increased and a slight change in conformation of HSA upon binding with PAEs. This observation again supports the MD simulation results as discussed earlier.

\subsection{The fluorescence quenching effect of PAEs on HSA}

HSA has only three intrinsic fluorophores: tryptophan (Trp), tyrosine (Tyr) and phenylalanine (Phe) residues (Xie et al., 2010). The intrinsic fluorescence of HSA is mainly contributed by the Trp residue, and the intensity is very sensitive to its microenvironment. Its intrinsic fluorescence would significant weak when the local surrounding of HSA was slightly altered.

In this research, the quenching mechanism was investigated by measuring the fluorescence spectra of HSA in the presence of different concentrations of PAEs at 291, 298 and $310 \mathrm{~K}$, respectively. These were corrected by subtracting the spectra of free PAEs from the quenching spectra according to relevant references (Soares et al., 2007). When the amount of the PAEs was added into HSA solution increased, the emission wavelength around $346 \mathrm{~nm}$ had a slight blue shift, with an intensity decreasing gradually. The results may be a binding of PAEs to HSA and a non-radiative energy transfer between them. The chromophore of HSA is possibly placed in a more hydrophobic environment.

The fluorescence quenching was analyzed by the Stern-Volmer equation (Lakowicz, 1999):

$F_{0} / F=1+K_{\mathrm{q}} \tau_{0}[Q]=1+K_{\mathrm{SV}}[Q]$ where $F_{0}$ and $F$ represent the steady-state fluorescence intensities in the absence and presence of quencher, respectively, $K_{\mathrm{SV}}$ is the Stern-Volmer quenching constant, $[Q]$ is the concentration of quencher, $\tau_{0}$ is the average lifetime of the molecule without any
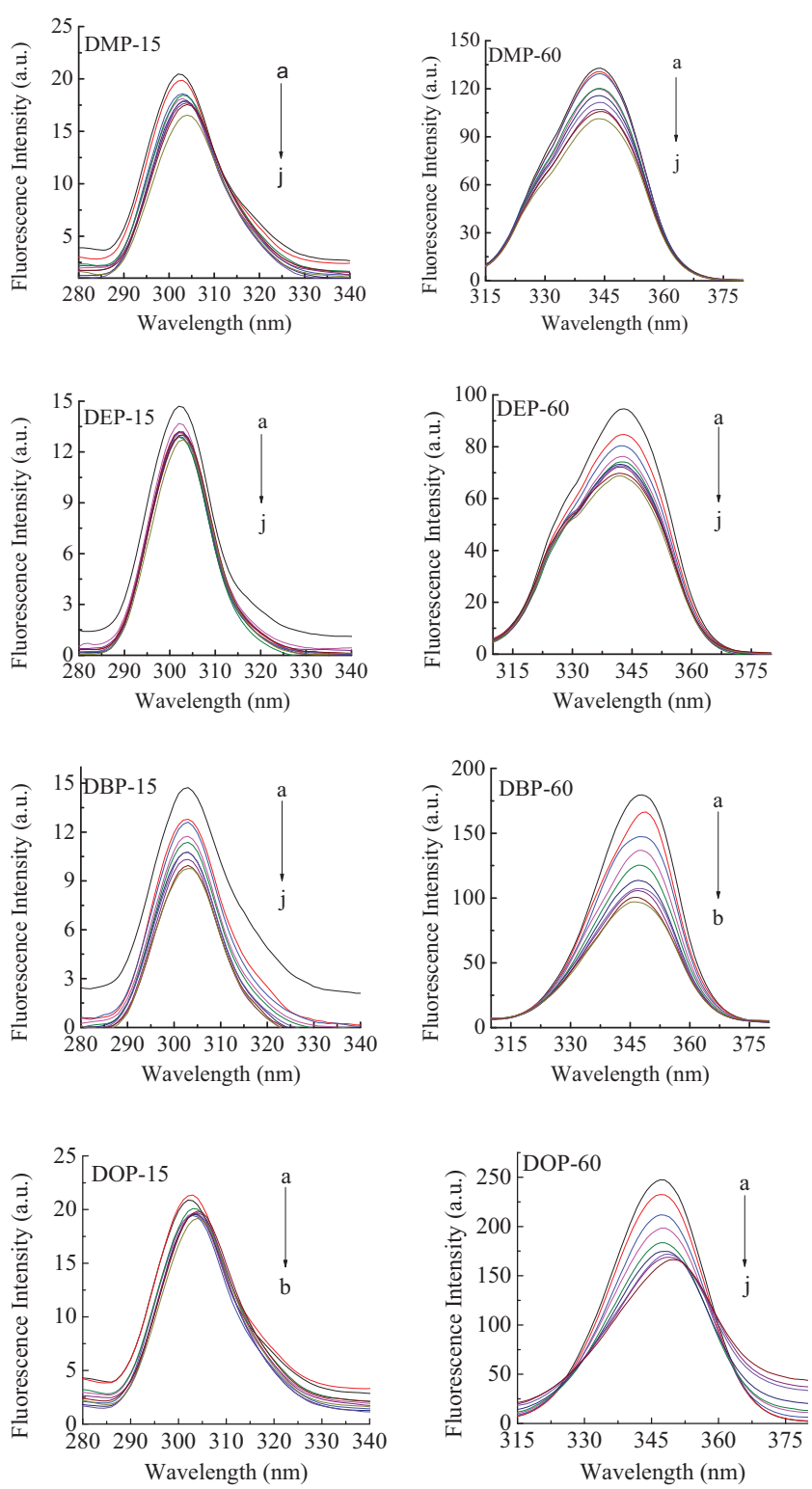

Fig. 3. Synchronous fluorescence spectrum of HSA ( $T=298 \mathrm{~K}, \mathrm{pH} 7.4)$. From a to $\mathrm{j}$ : $\mathrm{CHSA}=1 \mu \mathrm{M}$; $\mathrm{CPAEs}=0,10,20,30,40,50,60,70,80$ and $90 \mu \mathrm{M}$, respectively. (a) $\Delta \lambda=15 \mathrm{~nm}$ and (b) $\Delta \lambda=60 \mathrm{~nm}$. 
Table 1

Detail of estimation of four HSA-PAEs by FRET theory.

\begin{tabular}{lllll}
\hline Compound & $J\left(\mathrm{~cm}^{3} \mathrm{~L} \mathrm{~mol}^{-1}\right)$ & $E$ & $R_{0}(\mathrm{~nm})$ & $r(\mathrm{~nm})$ \\
\hline DMP & $2.55 \times 10^{-15}$ & 0.0099 & 2.033 & 4.376 \\
DEP & $4.49 \times 10^{-15}$ & 0.0165 & 2.233 & 2.925 \\
DBP & $2.68 \times 10^{-15}$ & 0.0670 & 2.049 & 3.174 \\
DOP & $9.61 \times 10^{-15}$ & 0.0328 & 1.180 & 2.065 \\
\hline
\end{tabular}

quencher and the fluorescence lifetime of the biopolymer is $10^{-8} \mathrm{~s}$ (Lakowicz and Weber, 1973), and $K_{\mathrm{q}}$ is the quenching rate constant of the biological macromolecule and equal to $K_{\mathrm{SV}} / \tau_{0}$. The maximum scattering collision quenching constant of various quenchers is $2 \times 10^{10} \mathrm{~L} /(\mathrm{mols})$ (Eftink and Ghiron, 1981), for the dynamic quenching.

The mechanism of quenching can be distinguished by the influencing effect of the temperature and viscosity and lifetime of the complex. Dynamic quenching constants are expected to be higher with increasing temperature, while static quenching lower. Table 1 lists the calculated $K_{\mathrm{SV}}$ and $K_{\mathrm{q}}$ values. It can be found that PAEs could effectively bind to HSA, and the binding constant decreases with the increase of temperature. The values of $K_{\mathrm{q}}$ are much larger than the maximum scattering collision quenching constant $2 \times 10^{10} \mathrm{~L} /(\mathrm{mol} \mathrm{s})$. It can be concluded that the quenching of HSA by PAEs were by the static quenching mechanism.

\subsection{Fluorescence resonance energy transfer}

The spectral studies suggested that HSA formed complex with PAEs. The molecular distance $(r)$ between HSA (donor) and PAEs (acceptor) can be calculated according to Förster's theory of dipole-dipole energy transfer. Generally, fluorescence resonance energy transfer (FRET) occurs whenever the emission spectrum of a fluorophore overlaps with the absorption spectrum of another molecule. The FRET was analyzed by Förster's non-radiative FRET theory. According to the equation:

$E=\frac{1-F}{F_{0}}=\frac{R_{0}^{6}}{\left(R_{0}^{6}+r^{6}\right)}$

$R_{0}^{6}=8.79 \times 10^{-25} K^{2} N^{-4} \varphi J$

$J=\left(\sum F(\lambda) \varepsilon(\lambda) \lambda^{4} \Delta \lambda\right) /\left(\sum F(\lambda) \Delta \lambda\right)$

where $r$ represents the distance between a donor and an acceptor, $R_{0}$ is the critical distance at which transfer efficiency equals to $50 \%, K^{2}$ is the orientation factor related to the geometry of the donor-acceptor dipole, $N$ is the refractive index of medium, $\varphi$ is the fluorescence quantum yield of the donor, $J$ expresses the degree of spectral overlap between the donor emission and the acceptor absorption, $F(\lambda)$ is the fluorescence intensity of the donor at
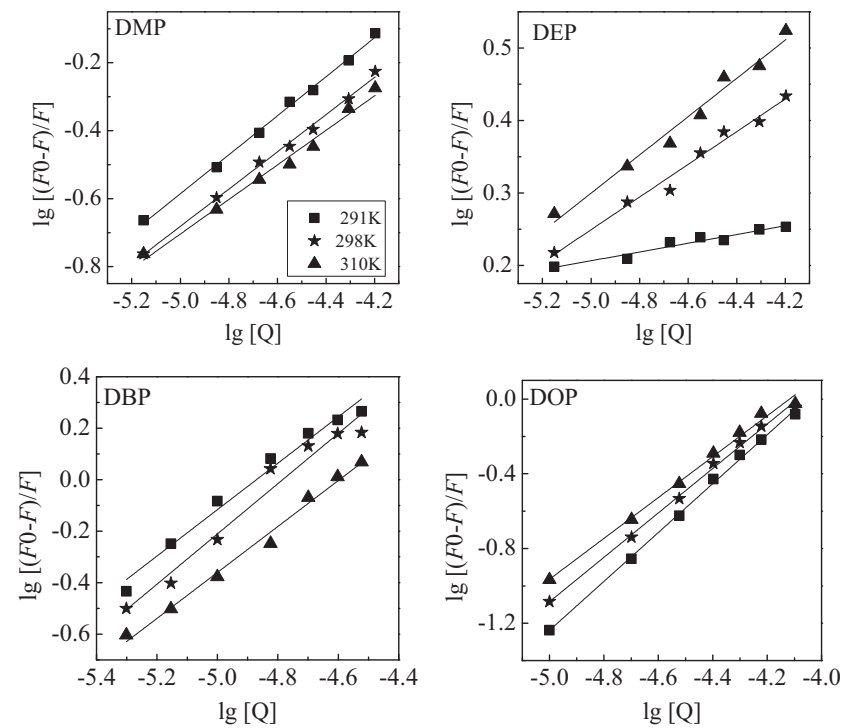

Fig. 4. The correspond to the Stern-Volmer curves for the quenching of HSA fluorescence by PAEs at three different temperatures.

wavelength range $\lambda$, and $\varepsilon(\lambda)$ is the molar absorption coefficient of the acceptor at wavelength $\lambda . K^{2}=2 / 3, N=1.36$, and $\varphi=0.15$. The calculated values of FRET efficiency $(E), J$ and $r$ are listed in Table 1. The distance between donor and acceptor was less than $7 \mathrm{~nm}$, indicating that the energy transfer from HSA to PAEs occurred with high possibility (Fani et al., 2012). This was in accordance with conditions of Förster theory of non-radioactive energy transfer and indicated a static quenching between PAEs and HSA.

\subsection{Binding constant and number of binding sites}

When small molecules binding independently to a set of equivalent sites of a macromolecule, the number of binding sites and the binding constant can be determined by the following equation (Zhao et al., 2006):

$\lg \left[\left(F_{0}-F\right) / F\right]=\lg K_{\mathrm{a}}+n \lg [Q]$

where $K_{\mathrm{a}}$ is the binding constant of drug and macromolecule, and $n$ is the number of binding sites. The $\lg [Q]$ versus $\lg \left[\left(F_{0}-F / F\right)\right]$ for the four PAEs was plotted in Fig. 4. The binding parameters for the interactions between PAEs and HSA were listed in Table 2.

The linearity of equation indicated that PAEs could bind to only one site on HSA (Table 2). We can see that the number of binding sites for HSA-DMP, HSA-DEP and HSA-DBP were lower than 1 , which implied that DMP, DEP and DBP were only partially bound to HSA. The order of binding affinity of HSA for all test phthalates

Table 2

The quenching constant and thermodynamic parameters of HSA-PAEs interaction at different temperatures.

\begin{tabular}{|c|c|c|c|c|c|c|}
\hline Compound & $T /(\mathrm{K})$ & $K_{\mathrm{a}} /\left(\mathrm{L} \mathrm{mol}^{-1}\right)$ & $n$ & $\Delta H^{\theta} /\left(\mathrm{kJ} \mathrm{mol}^{-1}\right)$ & $\Delta G^{\theta} /\left(\mathrm{kJ} \mathrm{mol}^{-1}\right)$ & $\Delta S^{\theta} /\left(\mathrm{J} \mathrm{mol}^{-1} \mathrm{~K}^{-1}\right)$ \\
\hline \multirow{4}{*}{ DMP } & 291 & $1.86 \times 10^{2}$ & 0.871 & \multirow{3}{*}{-37.94} & -12.64 & \multirow{3}{*}{-86.97} \\
\hline & 298 & $1.27 \times 10^{2}$ & 0.928 & & -12.00 & \\
\hline & 310 & $0.71 \times 10^{2}$ & 0.912 & & -10.99 & \\
\hline & 291 & $1.12 \times 10^{2}$ & 0.916 & \multirow{3}{*}{-19.45} & -25.53 & \multirow{4}{*}{-66.58} \\
\hline \multirow[t]{3}{*}{ DEP } & 298 & $0.62 \times 10^{2}$ & 0.970 & & -23.99 & \\
\hline & 310 & $0.65 \times 10^{2}$ & 0.905 & & -22.73 & \\
\hline & 291 & $2.58 \times 10^{4}$ & 0.906 & \multirow{3}{*}{-29.15} & -25.62 & \\
\hline \multirow[t]{3}{*}{ DBP } & 298 & $1.73 \times 10^{4}$ & 0.880 & & -24.18 & \multirow{3}{*}{-16.08} \\
\hline & 310 & $1.21 \times 10^{4}$ & 0.888 & & -24.24 & \\
\hline & 291 & $1.54 \times 10^{5}$ & 1.284 & \multirow{3}{*}{-45.90} & -27.61 & \\
\hline \multirow[t]{2}{*}{ DOP } & 298 & $6.10 \times 10^{4}$ & 1.173 & & -27.30 & \multirow[t]{2}{*}{-59.97} \\
\hline & 310 & $4.50 \times 10^{4}$ & 1.126 & & -28.90 & \\
\hline
\end{tabular}


Table 3

The binding parameters obtained from Surflex-Dock program.

\begin{tabular}{lllll}
\hline & Ligand & $-\log \left(K_{\mathrm{d}}\right)$ & $G /\left(\mathrm{kJ} \mathrm{mol}^{-1}\right)$ & Hydrogen bond \\
\hline \multirow{4}{*}{ Site I } & DMP & 4.64 & -26.48 & ARG218 \\
& DEP & 6.21 & -35.42 & ARG218 \\
& DBP & 7.97 & -45.50 & ARG218 \\
& DOP & 9.17 & -52.33 & LYS199, ARG218 \\
& DMP & 5.0070 & -28.58 & ASN391, ARG410, TYR411, SER489 \\
Site II & DEP & 5.3544 & -30.56 & ARG410, LYS414, SER489 \\
& DBP & 9.7897 & -55.88 & TYR411, LYS414, SER489 \\
& DOP & 13.4324 & -76.67 & ASN391, ARG410, YTR411, LYS414 \\
\hline
\end{tabular}

is: $\mathrm{DEP}>\mathrm{DMP}>\mathrm{DBP}>\mathrm{DOP}$. With increasing fatty chain lengths, $K_{\mathrm{a}}$ and $n$ value increase, which indicates high binding affinity could be formed between PAEs and HSA. PAEs are known as lipophilic compounds, which could insert into the hydrophobic cavity of the steric structure of HSA in aqueous solution. The PAEs have fatty chain, and the binding strength increases with the increase of the length of the fatty chain.

\subsection{Thermodynamic analysis and the nature of the binding force}

The interaction forces between small organic molecule and biological macromolecule includes hydrogen bond, Van der Waals force, hydrophobic force and electrostatic interactions. If the thermodynamic parameters enthalpy change $(\Delta H)$ does not vary much over the temperature range studied, then $\Delta H$ as well as $\Delta S$ can be evaluated from the Van't Hoff equation (Xie et al., 2010):

$\Delta G^{\theta}=-R T \ln K^{\theta}$

$\ln K^{\theta}=-\frac{\Delta H^{\theta}}{R T}+\frac{\Delta S^{\theta}}{R}$

The thermodynamic parameters, like enthalpy $\left(\Delta H^{\theta}\right)$, free energy change $\left(\Delta G^{\theta}\right)$ and entropy $\left(\Delta S^{\theta}\right)$ were evaluated according to references (Ross and Subramanian, 1981). The negative $\Delta H^{\theta}$ and $\Delta S^{\theta}$ values (Table 2) indicate that Van der Waals forces and hydrogen bonds play major role in the HSA-PAEs binding reaction (Table 3).

There are two high affinity binding sites for drugs in HSA, which can be marked by warfarin (site I) and ibuprofen (site II), respectively (Deeb et al., 2010). In order to identify the binding site of PAEs on HSA, site marker competitive experiments were carried out using these two probes. In the present experiment, PAEs were added to the solution of HSA which had been bound by site markers.

The binding constants of the four systems, which can be calculated by Stern-Volmer equation, were summarized as follow: $K$ (Ibuprofen, $1.16 \times 10^{5}, 1.18 \times 10^{5}, 1.18 \times 10^{5}$, $\left.1.17 \times 10^{5} \mathrm{~mol} / \mathrm{L}\right)<K\left(\right.$ Warfarin, $2.24 \times 10^{5}, 2.13 \times 10^{5}, 2.24 \times 10^{5}$, $\left.2.22 \times 10^{5} \mathrm{~mol} / \mathrm{L}\right)$. Obviously, the binding constant of the system with ibuprofen was almost $50 \%$ of that in the system without probe $\left(K=2.32 \times 10^{5}, 2.38 \times 10^{5}, 2.36 \times 10^{5}, 2.25 \times 10^{5} \mathrm{~mol} / \mathrm{L}\right)$, while the constant of the systems with and without warfarin had little difference. This demonstrates that ibuprofen could significantly affect the binding of PAEs to HSA, while warfarin had little influence. This indicated that the binding of PAEs to HSA is mainly located in site II (sub-domain IIIA) which is the same as Table 3.

\subsection{Molecular modeling study on the interaction between PAEs and HSA}

Molecular docking is an important method for understanding the binding properties (e.g. binding energy and binding site) of the protein are the structures of complexes of HSA and PAEs.
Based on the binding energy calculation, it is noticed that hydrophobic interactions and hydrogen bond are the major force in the binding model. The presence of hydrogen bond would decrease the hydrophilicity of the PAEs and increased their hydrophobicity, which made the HSA-PAEs complexes more stable.

As shown in Fig. 5 the PAEs molecule is situated within both binding sites in sub-domain IIIA. However, the binding of PAEs to site II produced lower free energy, which makes the binding more stable. And PAEs do prefer the binding site in sub-domain IIIA, demonstrating that binding by free energy is more stable than binding via interaction. Besides, the combination between PAEs molecules and HSA could also be attributed to hydrogen bond and the amino acid residues in the proximity of the ligand. These results correspond with those obtained by the experimental methods mentioned above. Therefore it can be concluded that the interaction between the PAEs and HSA is mainly hydrophobic, hydrogen bonding and Van der Waals forces.

\section{Discussion}

Now, there are more and more technologies and methods were used study between biological macromolecules and ligand, including spectroscopy, electrochemistry, NMR and mass spectrometry, etc. Meanwhile, computational simulation technology has made great development in the field of study of interaction in recent year. For example, imipenem binds to HSA at a high affinity site located in sub-domain IIIA and a low affinity site located in sub-domain IIAIIB by spectroscopic and computational approaches (Rehman et al., 2014).

However, each of these approaches has two aspects, which has its limits. Such as, although spectroscopy can be used to measure small molecules and biological macromolecular structure changes before and after the formation of complex information, but various spectroscopy methods application scope is limited, and need a variety of spectroscopy method. The electrochemical method that illustrated protein donates electron to ligands through charge transfer complexation (Balraj et al., 2012). However, it is difficult to determine some difficult oxidation of small molecules such as PBDEs. In NMR spectroscopy, such as nuclear Overhauser enhancement spectroscopy and correlation spectroscopy methods, they have been extensively used to study structural and dynamical properties of protein in solution. Pulse field gradient NMR is also an efficient method for detecting complexation of small molecules with their carrier proteins, like curcumin interacts with HSA (Singh et al., 2014). But its analysis of the sample amount is larger for large biological macromolecules has limitations and generally a costly.

In this context, to break through these limitations, need a variety of analysis methods of integrated applications and collaboration between different disciplines. For instance, the binding ability with bovine serum albumin as a model protein was investigated through proton magnetic resonance $\left({ }^{1} \mathrm{H}\right.$ NMR), ultraviolet visible spectroscopy (UV-vis), circular dichroism (CD), and fluorescence spectroscopy (Gao et al., 2006). This study combines the advantages of a variety of means to various interpretation of its action mechanism, including the role of proton transfer, before and after the change of the fluorescence quenching mechanism, the alpha helix and the strength of the affinity. On the other hand, it also depends on the continuous development of reasonable arrangement of various analytical techniques and perfect. Utilized reasonable optimization Settings using electrospray ionization mass spectrometry (ESI/MS) bovine serum albumin (BSA) was studied with 24 different bromine and hydroxyl replace the position of OH-PBDEs homologue of covalent complex interactions (Ma et al., 2014). 

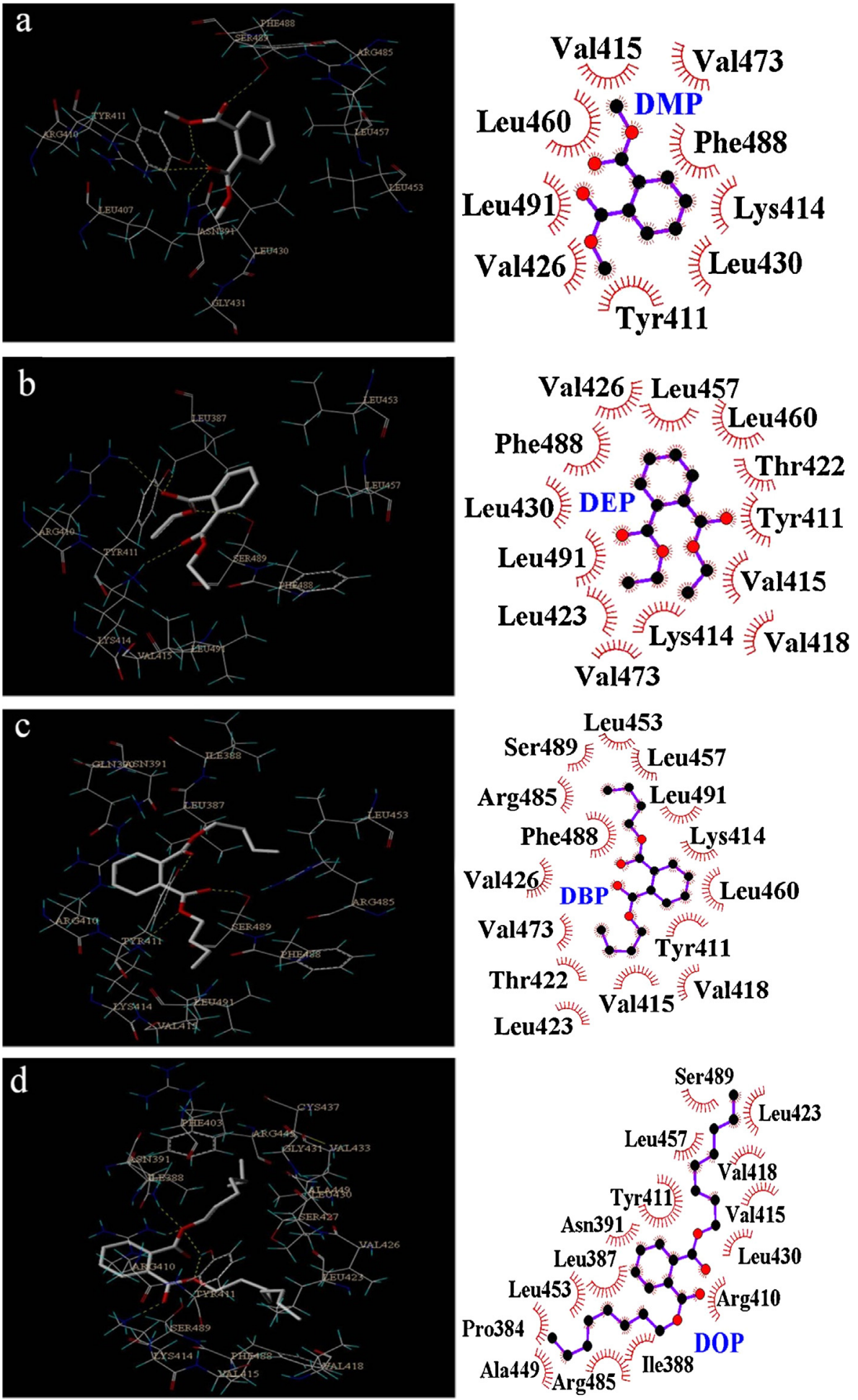

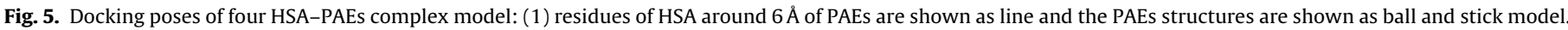
(2) The hydrophobic interactions were showed by Ligplot program.

\section{Conclusion}

In conclusion, the interaction between PAEs and HSA has been investigated by fluorescence, synchronous fluorescence, molecular docking and molecular dynamics simulation. According to experimental results, PAEs can bind with HSA during transport and metabolic processes in vitro. This will provide important insight into the interactions of the physiologically important protein HSA with endocrine disruptors PAEs and a theoretical basis for ecotoxicology and environmental risk assessment. Experimental results also shown that the binding of PAEs induced a conformational change of HSA, which was further proved by the analysis of molecular dynamics simulation.

\section{Transparency document}

The Transparency document associated with this article can be found in the online version. 


\section{Acknowledgements}

This work was kindly supported by National Natural Science Foundation of China, (21267008, 21167006); Guangxi Natural Science Foundation of China (2013GXNSF AA019034). The author also greatly thanks the help from Foundation of State Key Laboratory of Environmental Chemistry and Ecotoxicology from the Research Center for Eco-Environmental Sciences, Chinese Academy of Sciences (KF2011-21).

\section{References}

Balraj, C., Satheshkumar, A., Ganesh, K., Elango, K.P., 2012. Spectral, electrochemical and theoretical studies on the charge transfer complexes of azacyclonol with novel substituted 1,4-benzoquinones possessing tunable electron acceptor properties. RSC Adv. 2, 12384-12391.

Blair, J.D., Ikonomou, M.G., Kelly, B.C., Surridge, B., Gobas, F.A.P.C., 2009. Ultra-trace determination of phthalate ester metabolites in seawater, sediments, and biota from an urbanized marine inlet by LC/ESI-MS/MS. Environ. Sci. Technol. 43, 6262-6268.

Carter, D.C., Chang, B., Ho, J.X., Keeling, K., Krishnasami, Z., 1994. Preliminary crystallographic studies of four crystal forms of serum albumin. Eur. J. Biochem. 226, 1049-1052.

Deeb, O., Rosales-Hernández, M.C., Gómez-Castro, C., Garduño-Juárez, R., Correa-Basurto, J., 2010. Exploration of human serum albumin binding sites by docking and molecular dynamics flexible ligand-protein interactions. Biopolymers 93, 161-170.

Eftink, M.R., Ghiron, C.A., 1981. Fluorescence quenching studies with proteins. Anal. Biochem. 114, 199.

Fani, N., Bordbar, A., Ghayeb, Y., 2012. A combined spectroscopic, docking and molecular dynamics simulation approach to probing binding of a Schiff base complex to human serum albumin. Spectrochim. Acta A 103, 11-17.

Gao, H., Wang, Y.N., Fan, Y.G., Ma, J.B., 2006. Interactions of some modified monoand bis-beta-cyclodextrins with bovine serum albumin. Bioorg. Med. Chem. 14, 131-137.

Hansen, D.K., Grafton, T.F., 1994. Evaluation of di (2-ethylhexyl) phthalate-induced embryotoxicity in rodent whole-embryo culture. J. Toxicol. Environ. Health 43, 361-367.

Ibrahim, N., Ibrahim, H., Kim, S., Nallet, J.P., Nepveu, F., 2010. Interactions between antimalarial indolone-N-oxide derivatives and human serum albumin. Biomacromolecules 11, 3341-3351.

Jana, S., Ghosh, S., Dalapati, S., Guchhait, N., 2012. Exploring structural change of protein bovine serum albumin by external perturbation using extrinsic fluorescence probe: spectroscopic measurement, molecular docking and molecular dynamics simulation. Photochem. Photobiol. Sci. 11, 323-332.

Jiang, C.Q., Gao, M.X., He, J.X., 2002. Study of the interaction between terazosin and serum albumin: synchronous fluorescence determination of terazosin. Anal. Chim. Acta 452, 185-189.

Ko, S., Lee, W., Lee, S., Park, H., 2008. Nanosecond molecular dynamics simulations of Cdc25B and its complex with a 1,4-naphthoquinone inhibitor: implications for rational inhibitor design. J. Mol. Graph. Model. 27, 13-19.

Lakowicz, J.R., 1999. Instrumentation for fluorescence spectroscopy. Princ. Fluoresc. Spectrosc. 2, 25-61.
Lakowicz, J.R., Weber, G., 1973. Quenching of protein fluorescence by oxygen. Detection of structural fluctuations in proteins on the nanosecond time scale. Biochemistry 12, 4171-4179.

Li, J.Y., Zhu, X.L., Yang, C., Shi, R.W., 2010. Characterization of the binding of angiotensin II receptor blockers to human serum albumin using docking and molecular dynamics simulation. J. Mol. Model. 16, 789-798.

Liu, Y.P., Wang, S.H., Wang, L., 2013. Development of rapid determination of 18 phthalate esters in edible vegetable oils by gas chromatography tandem mass spectrometry. J. Agric. Food Chem. 61, 1160-1164.

Ma, Q., Bai, H., Wang, C., Xi, G.C., Zhang, Q., Meng, X.S., Chen, Y.X., Li, J.R., Ma, H.J. Guo, L.H., 2014. Investigation of noncovalent interactions between hydroxylated polybrominated diphenyl ethers and bovine serum albumin using electrospray ionization-ion mobility-mass spectrometry. Int. J. Mass Spectrom. 357, 34-44.

Mo, C.H., Cai, Q.Y., Tang, S.R., Zeng, Q.Y., Wu, Q.T., 2009. Polycyclic aromatic hydrocarbons and phthalic acid esters in vegetables from nine farms of the Rearl river delta, South China. Arch. Environ. Contam. Toxicol. 56, 181-189.

Pu, H.L., Jiang, H., Chen, R.R., Wang, H.C., 2014. Studies on the interaction between vincamine and human serum albumin: a spectroscopic approach. Luminescence 29, 471-479.

Qu, R.J., Feng, M.B., Sun, P., Wang, Z.Y., 2009. A comparative study on antioxidant status combined with integrated biomarker response in Carassius auratus fish exposed to nine phthalates. Environ. Toxicol. 30, 1125-1134.

Rehman, M.T., Shamsi, H., Khan, A.U., 2014. Insight into the binding mechanism of imipenem to human serum albumin by spectroscopic and computational approaches. Mol. Pharmacol. 11, 1785-1797.

Romani, F., Tropea, A., Scarinci, E., Federico, A., Russo, C.D., Lisi, L., Catino, S., Lanzone, A., Apa, R., 2014. Endocrine disruptors and human reproductive failure: the in vitro effect of phthalates on human luteal cells. Fertil. Steril, 102, 831-837.

Ross, P.D., Subramanian, S., 1981. Thermodynamics of protein association reactions: forces contributing to stability. Biochemistry 20, 3096-3102.

Singh, D.V., Bharti, S.K., Agarwal, S., Roy, R., Misra, K., 2014. Study of interaction of human serum albumin with curcumin by NMR and docking. J. Mol. Model. 20, 2365.

Soares, S., Mateus, N., de Freitas, V., 2007. Interaction of different polyphenols with bovine serum albumin (BSA) and human salivary alpha-amylase (HSA) by fluorescence quenching. J. Agric. Food Chem. 55, 6726-6735.

Suzuki, T., Yaguchi, K., Suzuki, S., Suga, T., 2001. Monitoring of phthalic acid monoesters in river water by solid-phase extraction and GC-MS determination. Environ. Sci. Technol. 35, 3757-3763.

Wardell, M., Wang, Z.M., Ho, J.X., Robert, J., Ruker, F., Ruble, J., Carter, D.C., 2002. The atomic structure of human methemalbumin at 1.9 angstrom. Biochem. Biophys. Res. Commun. 291, 813-819.

Xie, X.Y., Wang, X.R., Xu, X.M., Sun, H.J., Chen, X.G., 2010. Investigation of the interaction between endocrine disruptor bisphenol A and human serum albumin. Chemosphere 80, 1075-1080.

Xie, X.Y., Wang, Z.W., Zhou, X.M., Wang, X.R., Chen, X.G., 2011. Study on the interaction of phthalate esters to human serum albumin by steady-state and time-resolved fluorescence and circular dichroism spectroscopy. J. Hazard. Mater. 192, 1291-1298.

Yamasaki, K., Maruyama, T., Kragh-Hansen, U., Otagiri, M., 1996. Characterization of site I on human serum albumin: concept about the structure of a drug binding site. Biochim. Biophys. Acta 1295, 147-157.

Zhao, H.W., Ge, M., Zhang, Z.X., Wang, W.F., Wu, G.Z, 2006. Spectroscopic studies on the interaction between riboflavin and albumins. Spectrochim. Acta A 65, $811-817$.

Zhou, X.M., Lü, W.J., Su, L., Shan, Z.J., Chen, X.G., 2012. Binding of phthalate plasticizers to human serum albumin in vitro: a multispectroscopic approach and molecular modeling. J. Agric. Food Chem. 60, 1135-1145. 